\title{
Piecewise Linear Weighted Iterative Algorithm for Beam Alignment in Scanning Beam Interference Lithography
}

\author{
Ying SONG*, Bayanheshig*, Shuo LI, Shan JIANG, and Wei WANG \\ National Engineering Research Centre for Diffraction Gratings Manufacturing and Application, Changchun Institute of \\ Optics, Fine Mechanics and Physics, Chinese Academy of Sciences, Jilin 130033, China \\ ${ }^{*}$ Corresponding authors: Ying SONG and Bayanheshig $\quad$ E-mail: songyingtec@126.com; bayin888@sina.com
}

\begin{abstract}
To obtain a good interference fringe contrast and high fidelity, an automated beam iterative alignment is achieved in scanning beam interference lithography (SBIL). To solve the problem of alignment failure caused by a large beam angle (or position) overshoot exceeding the detector range while also speeding up the convergence, a weighted iterative algorithm using a weight parameter that is changed linearly piecewise is proposed. The changes in the beam angle and position deviation during the alignment process based on different iterative algorithms are compared by experiment and simulation. The results show that the proposed iterative algorithm can be used to suppress the beam angle (or position) overshoot, avoiding alignment failure caused by over-ranging. In addition, the convergence speed can be effectively increased. The algorithm proposed can optimize the beam alignment process in SBIL.
\end{abstract}

Keywords: Piecewise linear weighted iterative algorithm; beam alignment; scanning beam interference lithography (SBIL); overshoot suppression; convergence speed

Citation: Ying SONG, Bayanheshig, Shuo LI, Shan JIANG, and Wei WANG, "Piecewise Linear Weighted Iterative Algorithm for Beam Alignment in Scanning Beam Interference Lithography," Photonic Sensors, 2019, 9(4): 344-355.

\section{Introduction}

Periodic micro/nanostructures have numerous applications, such as photonic crystals [1], liquid crystal displays [2], solar cells [3, 4], high density data storage [5, 6], and biosensors [7, 8]. A number of technologies have been utilized to fabricate periodic structures, including interference lithography [9], nanoimprinting [10, 11], electron beam lithography $[12,13]$, focused ion beam etching [14], and direct laser writing [15-17]. These commonly used techniques are difficult to produce large area micro/nanostructures with diameters of several hundred millimeters. Scanning beam interference lithography (SBIL) is a technique for fabricating periodic patterns over a large area with high phase fidelity. In SBIL, two small-diameter beams are used to generate an interference image that is exposed onto a photoresist on a substrate that is step-scanned under the interference image by a high performance stage $[18,19]$.

To obtain a good interference fringe contrast and low accumulated phase error, the tolerances for the incident angle difference and position overlaps in the two arms are of the order of $\mu \mathrm{rad}$ and $\mu \mathrm{m}$, respectively. An automated beam alignment system consisting of two tip-tilt mirrors and two position sensing detectors (PSDs) is used to achieve tight tolerances. Beam alignment operated before the exposure is based on an iterative algorithm, which 
can satisfy the speed and accuracy requirements of SBIL [20-22]. Certain real-time feedback algorithms are not implemented in the beam alignment system of SBIL [23, 24].

The Massachusetts Institute of Technology (MIT) firstly proposed an effective iterative algorithm for beam alignment in its SBIL system "nanoruler" $[18-21,25]$. We call this the "original iterative algorithm". Nevertheless, if the initial beam deviation is large or the optical parameters are inappropriate, the beam angle or position overshoots may be very large at the beginning of the alignment process. The large overshoot may cause the spot position to exceed the measuring range of the detectors. This finding indicates that the alignment has failed. In our previous paper [25], we discussed the possible cases causing the alignment failure and provided a simple weighted iterative algorithm to reduce the overshoot automatically. In this algorithm, a constant weight factor between 0 and 1 is used to suppress the overshoot by sacrificing convergence speed, and the smaller the overshoot is, the slower the convergence is. A combined two-stage weighted iterative algorithm, adopting a simple weighted iterative algorithm at the beginning stage and original iterative algorithm at the subsequent stage, can increase the convergence speed to a certain extent. However, the convergence speed is still lower than that by the original algorithm.

In this paper, a general mathematical model for the weighted iterative algorithm is given. The value ranges for the weight factors are determined by convergence property analysis. A piecewise linear weighted (PLW) iterative algorithm is then proposed. The variation in beam angle and position deviation with different iterative algorithms is compared by simulation and experiment. The results show that the proposed iterative algorithm can increase the convergence speed effectively and maintain the overshoot at a low level to prevent alignment failure caused by the spot missing the PSDs.

\section{Principle and analysis}

\subsection{General model of the weighted iterative algorithm}

The beam alignment system in SBIL is shown in Fig. 1. M1 and M2 are the tip-tilt mirrors. M1 is located further from the position decoupling plane (PDP). M1 influences the beam position more than M2 when they tilt at the same angle. Hence, the beam is aligned by alternately using M1 to align the position and M2 to align the angle. For the decoupling optical system, only the translation at the PDP can be sensed by one PSD (called position PSD, PPSD) with the proportion factor $K_{\mathrm{pos}}=1-L_{1} / f_{1}$. The beam angle at the angle decoupling plane (ADP) is converted proportionally to the spot location on another PSD (called angle PSD, APSD). The proportion factor $K_{\text {angle }}$ numerically equals $f_{2}$ [20]. If the two beams are aligned according to the decoupling planes, they have the same position and angle at any point in the optical path after M2. In this paper, the beam "position" and "angle" refer to those at the decoupling planes.

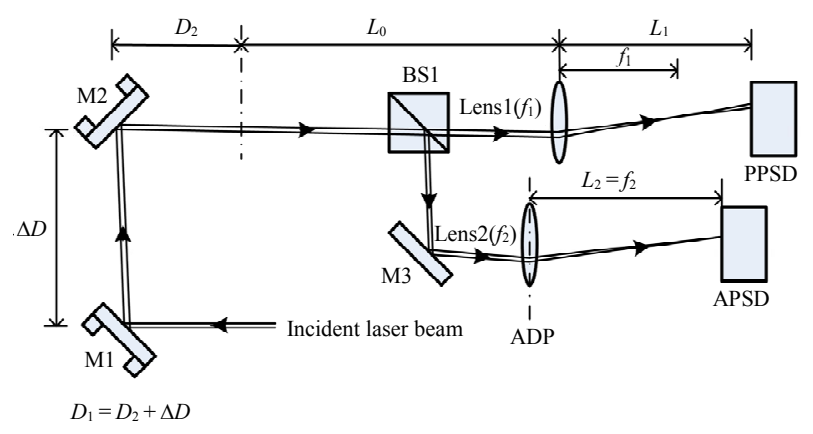

Fig. 1 Optical layout of the automated beam alignment system used for SBIL.

The beam angle and position can be aligned both in the $X$ and $Y$ directions. Taking one-dimensional beam alignment for illustration, with the other being similar, each iteration in the weighted iterative algorithm contains two steps. In the first step of an $n$th iteration, M1 tilts to reduce the position deviation $k_{p} p_{(n-1,2)} . k_{p}$ is a weight factor, and $p_{(n-1,2)}$ is the position deviation after the 2nd step of the $(n-1)$ th iteration. In the following step, one can 
reduce the angle deviation $k_{a} a_{(n, 1)}$ by actuating M2. $k_{a}$ is another weight factor, and $a_{(n, 1)}$ is the angle deviation after the first step of the $n$th iteration. Suppose the initial position deviation is $p_{0}$ from the desired position, and the angle deviation is $a_{0}$. The weighted iteration can be described by a matrix as (1) and (2)

$$
\begin{gathered}
{\left[\begin{array}{l}
p_{(n, 1)} \\
a_{(n, 1)}
\end{array}\right]=\mathbf{A}\left[\begin{array}{l}
p_{(n-1,2)} \\
a_{(n-1,2)}
\end{array}\right]=\mathbf{A}(\mathbf{B A})^{n-1}\left[\begin{array}{l}
p_{0} \\
a_{0}
\end{array}\right]} \\
{\left[\begin{array}{l}
p_{(n, 2)} \\
a_{(n, 2)}
\end{array}\right]=\mathbf{B}\left[\begin{array}{l}
p_{(n, 1)} \\
a_{(n, 1)}
\end{array}\right]=(\mathbf{B A})^{n}\left[\begin{array}{l}
p_{0} \\
a_{0}
\end{array}\right]}
\end{gathered}
$$

where $\mathbf{A}=\left[1-k_{p} 0 ;-k_{p} / D_{1} 1\right]$ and $\mathbf{B}=\left[1-k_{a} D_{2}\right.$; $\left.01-k_{a}\right] . p_{(n, 1)}$ and $a_{(n, 1)}$ are the position and angle deviations after the first step of the $n$th iteration, respectively, and $p_{(n, 2)}$ and $a_{(n, 2)}$ are those after the second step of the $n$th iteration. The position deviation is acquired by a position PSD, and the angle deviation is acquired by an angle PSD.

During the alignment process, the angle overshoot relative to the reference angle is defined as $a_{(1,1)}$, and the position overshoot is $p_{(1,2)}$. Equation (1) and (2) show $a_{(1,1)}=-k_{p} p_{0} / D_{1}+a_{0}$ and $p_{(1,2)}=\left(1-k_{p}\right) p_{0}+k_{a} D_{2}\left(k_{p} p_{0} / D_{1}-a_{0}\right)$. The original iterative algorithm proposed by MIT is a special case of the weighted iterative algorithm when $k_{p}=k_{a}=1$. In this case, if $a_{(1,1)}$ is considerably larger than $a_{0}$, the spot in the APSD is likely to exceed the measuring range of the detector. This causes the iterative process to fail in the first iteration. A similar situation occurs on the PPSD if $p_{(1,2)}$ is large. To solve the problem, a simple weighted iterative algorithm is proposed in a previous paper. We choose a constant $k_{p}\left(k_{p}<1\right)$ and $k_{a}=1$ to reduce the angle overshoot as $k_{p}$ has greater influence on the angle deviation. Similarly, $k_{a}<1$ and $k_{p}=1$ can be used to suppress the position overshoot [25]. The angle or position overshoot are reduced at the expense of a low convergence speed. The circumstance of the angle overshoot and position overshoot both large is uncommon, unless the optical parameters are very inappropriate (e.g. $\left.D_{2} / D_{1} \approx 1\right) . k_{a}<1$ and $k_{p}<1$ can be chosen to suppress the angle overshoot and position overshoot simultaneously.

\subsection{Convergence property analysis}

The convergence property will be divided into two cases of $k_{p} \neq 1, k_{a}=1$, and $k_{a} \neq 1, k_{p}=1$ to be discussed separately. When $k_{p} \neq 1$ and $k_{a}=1$, we can obtain (3)-(6) by matrix operation

$$
\mathbf{A}(\mathbf{B A})^{n-1}=\left\{\begin{array}{l}
\mathbf{A}, n=1 \\
m_{p}{ }^{n-2} \mathbf{A B A}, n \geq 2
\end{array}\right.
$$

$$
(\mathbf{B A})^{n}=m_{p}{ }^{n-1}(\mathbf{B A})
$$

$$
\begin{gathered}
\mathbf{A B A}=\left[\begin{array}{cc}
\left(1-k_{p}\right) m_{p} & -\left(1-k_{p}\right) D_{2} \\
-k_{p} m_{p} / D_{1} & k_{p} D_{2} / D_{1}
\end{array}\right] \\
\mathbf{B A}=\left[\begin{array}{cc}
m_{p} & -D_{2} \\
0 & 0
\end{array}\right]
\end{gathered}
$$

where $m_{p}=\left(1-k_{p}\right)+k_{p} D_{2} / D_{1}$.

To ensure convergence for the alignment process, $0 \leq\left|m_{p}\right|<1$ is required. If $k_{p}=D_{1} /\left(D_{1}-D_{2}\right)$ and $m_{p}=0$, the convergence becomes the fastest. Equations (3) and (4) show the alignment end when $n \geq 3$, and $\left[p_{(n, 1)}, a_{(n, 1)}\right]^{\mathrm{T}}=\left[p_{(n, 2)}, a_{(n, 2)}\right]^{\mathrm{T}}=0(n \geq 3)$, which means that any accuracy requirements can be satisfied in theory. However, if $D_{1}$ is close to $D_{2}$, the angle overshoot $a_{(1,1)}=-k_{p} p_{0} / D_{1}+a_{0}=-p_{0} /\left(D_{1}-D_{2}\right)+a_{0}$ will be very large. The fast convergence speed is gained at the sacrifice of overshoot.

The iterations with the same $\left|m_{p}\right|$ have the similar convergence speed. Each nonzero $\left|m_{p}\right|$ value can give two solution $k_{p}$ 's in the domains of $0<k_{p}<D_{1} /$ $\left(D_{1}-D_{2}\right)$ and $D_{1} /\left(D_{1}-D_{2}\right)<k_{p}<2 D_{1} /\left(D_{1}-D_{2}\right)$. According to the expression of $a_{(1,1)}$, a large $k_{p}$ is not a good choice because it may lead to a large $a_{(1,1)}$ when $p_{0}$ and $a_{0}$ have opposite signs. Therefore, $\left(0, D_{1} /\left(D_{1}-D_{2}\right)\right]$ is a better value range for $k_{p}$, and correspondingly, $0 \leq m_{p}<1$.

When $k_{a} \neq 1$ and $k_{p}=1$, the matrices become (7)-(9)

$$
\begin{aligned}
& \mathbf{A}(\mathbf{B A})^{n-1}=m_{a}{ }^{n-1} \mathbf{A} \\
& (\mathbf{B A})^{n}=m_{a}{ }^{n-1}(\mathbf{B A})
\end{aligned}
$$




$$
\mathbf{B A}=\left[\begin{array}{cc}
k_{a} D_{2} / D_{1} & -k_{a} D_{2} \\
-\left(1-k_{a}\right) / D_{1} & 1-k_{a}
\end{array}\right]
$$

where $m_{a}=\left(1-k_{a}\right)+k_{a} D_{2} / D_{1}$. This finding is similar to the results of the analysis above. The domain $\left(0, D_{1} /\left(D_{1}-D_{2}\right)\right]$ is a good choice for $k_{a}$, and correspondingly, $0 \leq m_{a}<1$.

\subsection{Piecewise linear weighted (PLW) iterative algorithm}

The value range for $k_{p}$ and $k_{a}$ is limited to $(0,1)$ in previous iterative algorithms. In the PLW iterative algorithm, the value range for the weight factors is extended to $\left(0, D_{1} /\left(D_{1}-D_{2}\right)\right]$, and the key weight factor is changed piecewise linearly with different slopes in different segments. The segments are divided according to the angle or position deviation.

We take the angle overshoot suppression in the $X$ direction as an example, with other cases being similar. The weight factor $k_{a}$ is kept at 1 constantly, and $k_{p}, \min \leq k_{p} \leq k_{p, \max }$, where $0<k_{p, \min }<k_{p, \max } \leq$ $D_{1} /\left(D_{1}-D_{2}\right)$. In the first iteration, $k_{p(1)}=k_{p, \text { min. }}$. When $n \geq 2$, the angle deviation is set to $m+1$ segments with $m$ angle thresholds $\xi_{a, 1}, \xi_{a, 2}, \cdots, \xi_{a, m}\left(\xi_{a, 1}>\right.$ $\left.\xi_{a, 2}>\cdots>\xi_{a, m}\right) . k_{p}$ is changed linearly with a different slope in different segments. When the local maximum $a_{(n, 1)}$ is less than the smallest threshold $\xi_{a, m}, k_{p(n)}$ equals $k_{p, \max }$. The changes in $k_{p}$ can be described by (10)

$$
k_{p(n)}=\left\{\begin{array}{l}
k_{p, \min }, n=1 \\
\min \left(k_{p(n-1)}+\Delta_{1}, k_{p, \max }\right), \xi_{a, 1} \leq a(n, 1) \& n \geq 2 \\
\vdots \\
\min \left(k_{p(n-1)}+\Delta_{i+1}, k_{p, \max }\right), \xi_{a, i+1} \leq a(n, 1)<\xi_{a, i} \& n \geq 2 \\
\vdots \\
\min \left(k_{p(n-1)}+\Delta_{m}, k_{p, \max }\right), \xi_{a, m} \leq a(n, 1)<\xi_{a, m-1} \& n \geq 2 \\
k_{p, \max }, a(n, 1)<\xi_{a, m} \& n \geq 2
\end{array}\right.
$$

where $k_{p(n)}$ is the $k_{p}$ value in the $n$th iteration. $\Delta_{i}$ is the $k_{p}$ increment in the $i$ th $(i=1,2, \cdots, m)$ segment, and $\Delta_{1}<\Delta_{2} \cdots<\Delta_{m-1}<\Delta_{m}$. The differential value is fixed in each segment and increased gradually in different segments. The $k_{p}$ increases from $k_{p}$, min to $k_{p, \max }$ with a decrease of $a_{(n, 1)}$.

Compared with the simple weighted iterative algorithm, the value range of $k_{p}$ is extended. If one chooses a small $k_{p, \min }$ in the first iteration, the angle overshoot $a_{(1,1)}$ can be significantly suppressed. In the middle part of the iteration, $k_{p}$ is gradually increased and it is different in each iteration. Each iteration can be regarded as a small segment, and $k_{p}$ is constant in each segment. Smoothly switching $k_{p}$ leads to a smooth convergence. An appropriate $\Delta_{i}$ can maintain a fast convergence speed. When $a_{(n, 1)}$ is sufficiently small $\left[a_{(n, 1)}<\xi_{a, m}\right]$, the angle deviation converges to zero as soon as possible. The convergence is faster, and the angle deviations maintain a low level.

\section{Experiments}

The experimental optical layout is shown in Fig. 2, where BS1 - BS3 are the beam splitters, and M1, M2, and M5 are the planar mirrors. M3 and M4 are tip-tilt mirrors. The decoupling topology is similar to that in Fig. 1. To realize the alignment of two beams, the beam from the laser source is split into a reference beam and a regulating beam. Before the beam alignment, the regulating beam is blocked by a shutter (S1). As shown in Fig. 2(a), the reference beam passes through M1, M2, and BS2 before entering the decoupling topology. The spot locations at the APSD and PPSD are recorded and converted to a beam position and angle by the computer program, which are treated as alignment targets. Afterwards, the reference beam is blocked by another shutter (S2), and S1 is removed to let out the regulating beam. As shown in Fig. 2(b), the regulating beam goes through $\mathrm{M} 3, \mathrm{M} 4$, and BS2 
before entering the same decoupling topology. The regulating beam is aligned to the targets by tilting M3 and M4. BS2 is located at the PDP in the experiment, and a screen is placed near BS2 as shown in Fig. 2. The spot of the reflected light of the reference beam (the spot of the reference beam) and the spot of the transmitted light of the regulating beam (the spot of the regulating beam) through BS2 will appear on the screen when S1 and S2 are removed after the alignment. The beam alignment on the PDP can be roughly observed by the spots on the screen with the naked eye.

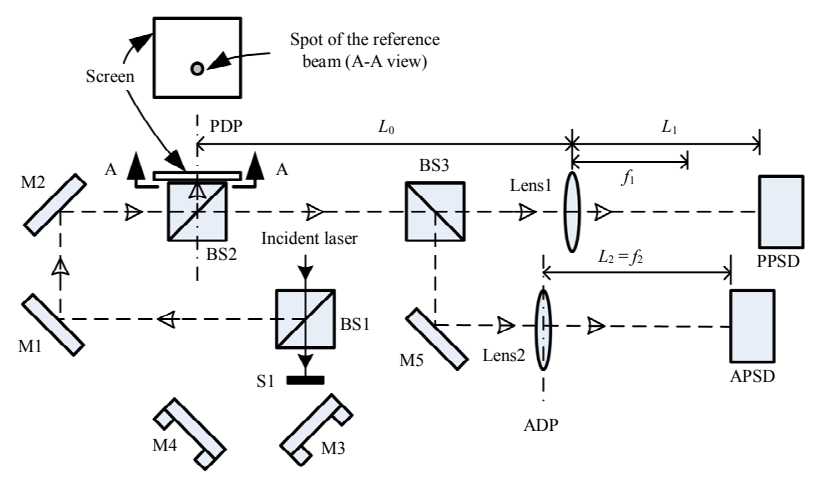

(a)

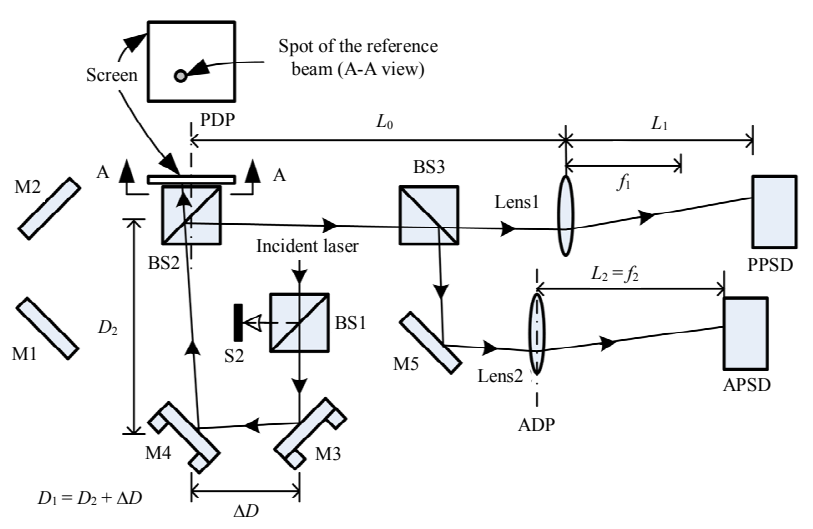

(b)

Fig. 2 Experimental optical layout: (a) the reference beam path and (b) the regulating beam path.

A $4.7 \mathrm{~mW}$ laser diode module with a wavelength of $532 \mathrm{~nm}$ (model CPS532, Thorlabs) is used as a laser source. M3 and M4 are the planar mirrors fixed onto precise mounts (model 8816-6, Newport) with two picomotors used to provide tip and tilt adjustment. A PSD with a sensor size of $9 \mathrm{~mm} \times$ $9 \mathrm{~mm}$ and a resolution of $\pm 0.2 \mu \mathrm{m}$ for $\mathrm{a} \pm 2 \mathrm{~mm}$ dynamic range (model OPB-U-9H, Newport) is used as a PPSD. The APSD located at the focus plane of Lens2 has a sensor size of $4 \mathrm{~mm} \times 4 \mathrm{~mm}$ and a resolution of $\pm 0.1 \mu \mathrm{m}$ for a $\pm 1 \mathrm{~mm}$ dynamic range (model OPB-U-4H, Newport). The optical parameters are $f_{1}=250 \mathrm{~mm}, L_{1}=400 \mathrm{~mm}, f_{2}=400 \mathrm{~mm}$, $D_{1}=590 \mathrm{~mm}$, and $D_{2}=440 \mathrm{~mm}$. We can obtain the proportion factor of the decoupling system $K_{\text {pos }}=$ $1-L_{1} / f_{1}=-0.6$ and $K_{\text {angle }}=0.4 \mu \mathrm{m} / \mu \mathrm{rad}$. The distance between Lens1 and the PDP is $L_{0}=L_{1} /\left|K_{\mathrm{pos}}\right|=$ $666.7 \mathrm{~mm}$.

An automatic program running on a computer is developed with Labview. The program acquires the readings from the PSD through a universal serial bus (USB) interface and adjusts two tip-tilt mirrors through an ethernet interface. After the optical system is accomplished, the reference beam is blocked, and the regulating beam is let out. The program makes only one picomotor linear step, and the other picomotors are kept stationary. In the meantime, the changes in the beam position and angle are recorded. Then, the relationship between the picomotor's movements and the beam parameter (position and angle) changes can be acquired online by one-order polynomial fitting. After moving every picomotor in the two tip-tilt mirror mounts independently, we can obtain the complete system characteristics for the actuators and beam parameter changes. Due to the nonlinearity of the picomotor, the relationships are slightly different when the picomotor's step numbers or the movement direction are different. The experimental results show that the influence of the nonlinearity is small. Before the alignment, the program records the position and angle of the reference beam as an alignment target. Afterwards, the regulating beam is aligned automatically to the targets based on the iterative algorithm used by the program.

Besides the experiment, the angle and position deviation changes are simulated with the same initial conditions and parameters as those in the experiment using Matlab. The consistency between the experiment and simulation can be checked. 


\section{Results}

We take alignment in the $X$ direction for illustration and aim to reduce the angle overshoot, which is the same situation as that in the analysis above. To compare different iterative algorithms, the initial deviations $a_{0}$ and $p_{0}$ should be adjusted to similar values. So, M3 and M4 move certain steps in the $X$ direction after the end of the previous alignment. When M3 moves +2000 steps and M4 moves -2600 steps, $a_{0} \approx 260 \mu \mathrm{rad}$, and $p_{0} \approx-400 \mu \mathrm{m}$. The initial deviation is chosen small values to avoid the possible alignment failure when the original iterative algorithm is used. The alignment tolerances are set to $20 \mu \mathrm{rad}$ (angle) and $20 \mu \mathrm{m}$ (position). In the second step of an $n$th iteration, we check whether the accuracy requirements are satisfied.

Figure 3 shows the changes in the position and angle deviations using the original iterative algorithm. In the whole iterative process, the weight parameters $k_{p}$ and $k_{a}$ are held at a constant value of 1 . The experimental results agree with the simulation, and the small differences between the experiment and simulation are caused by the nonlinearity of the picomotors. The angle overshoot is approximately $-850 \mu \mathrm{rad}$, and the iteration tolerances are satisfied when the number of iteration is $n \geq 11$.

The alignment results with the combined two-stage weighted iterative algorithm are shown in Fig. 4. At the beginning of the alignment, a small value for $k_{p}$ is chosen $\left(k_{p}=0.5\right)$ to suppress the angle overshoot. When the angle deviation is smaller than $\xi_{a, 1}, k_{p}$ is changed to 1 to increase the convergence speed. Let $\xi_{a, 1}=350 \mu \mathrm{rad}$, the combined two-stage weighted iterative algorithm is described by (11). As shown in Fig. 4, the angle overshoot is suppressed to $-628 \mu \mathrm{rad}$, but the convergence speed is slower than that observed with the original iterative algorithm for $n \geq 13$ for satisfying the accuracy requirements.

$$
k_{p}(n)=\left\{\begin{array}{l}
0.5, a(n, 1) \geq \xi_{a, 1} \\
1, a(n, 1)<\xi_{a, 1} .
\end{array}\right.
$$

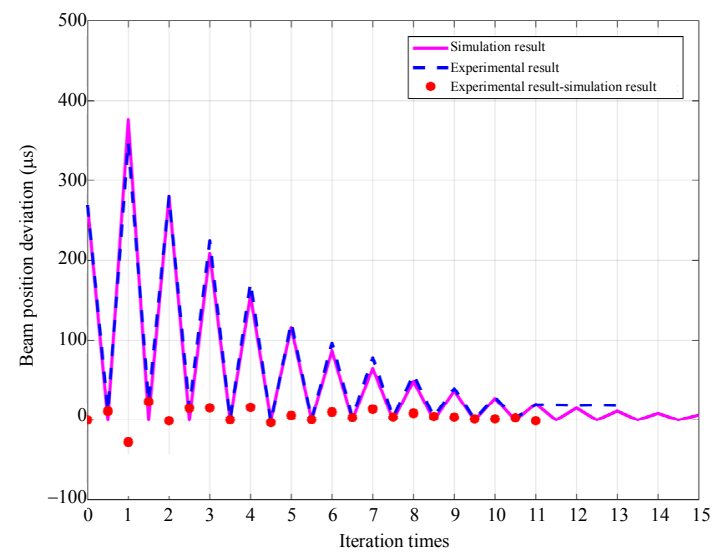

(a)

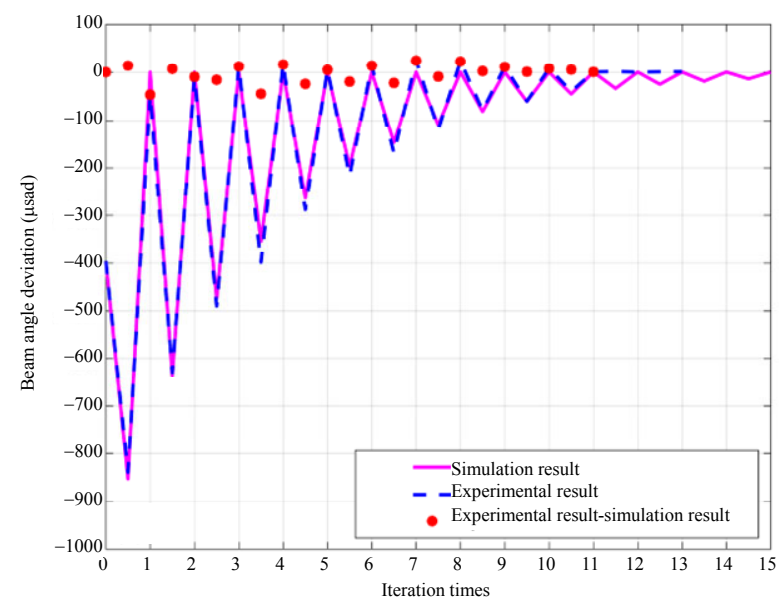

(b)

Fig. 3 Experimental and simulation results for beam alignment with the original iterative algorithm: (a) and (b) show the beam position and angle deviations, respectively.

The PLW iterative algorithm is shown as (10). In the experiment, the first group of parameters (parameters \#1) chosen are $m=2, \xi_{a, 1}=350 \mu \mathrm{rad}$, $\xi_{a, 2}=200 \mu \mathrm{rad}, k_{p, \min }=0.5$, and $k_{p, \max }=D_{1} /\left(D_{1}-D_{2}\right)-$ $0.05=3.88$. The increments in the two segments are $\Delta_{1}=0.2$ and $\Delta_{2}=0.4$, respectively. The changes in the position and angle deviations are shown in Figs. 5(a) and 5(b). Compared with the combined two-stage weighted iterative algorithm, $k_{p}$ is both 0.5 when $n=$ 1. Therefore, the angle overshoot in Fig. 4(b) is close to that in Fig. 5(b). For increasing $k_{p}$, the convergence is faster compared with the combined iterative algorithm and the original iterative algorithm. The iteration is completed when $n=9$. With the PLW iterative algorithm, a low angle overshoot is acquired, and the convergence speed is meanwhile effectively boosted. 


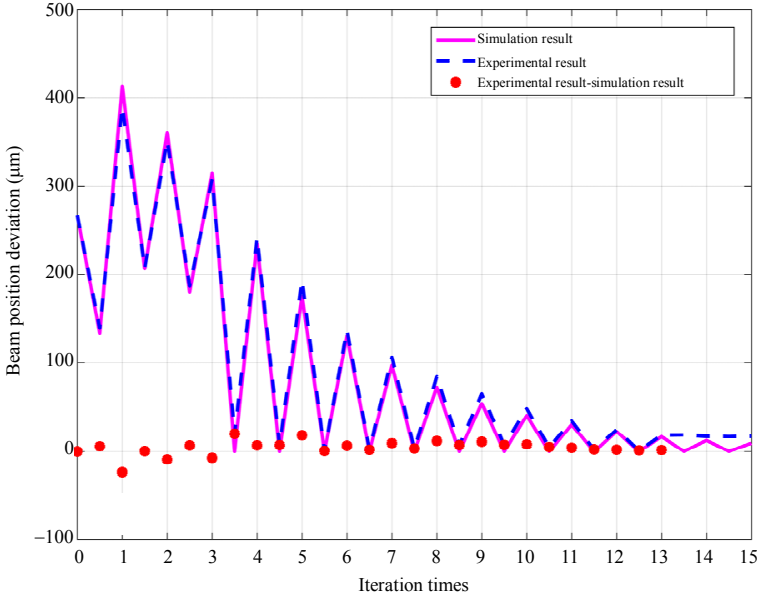

(a)

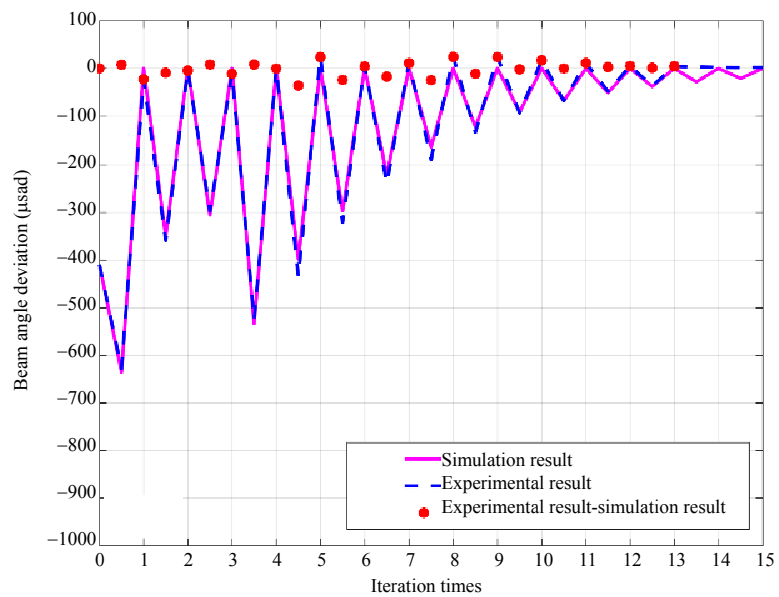

(b)

Fig. 4 Experimental and simulation results for the beam position and angle deviation changes with the combined two-stage iterative algorithm: (a) and (b) show the beam position and angle deviations, respectively.

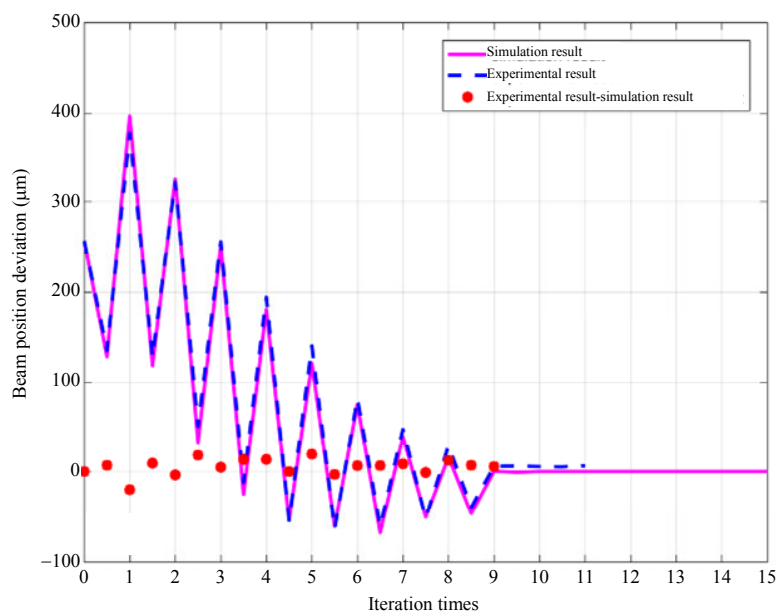

(a)

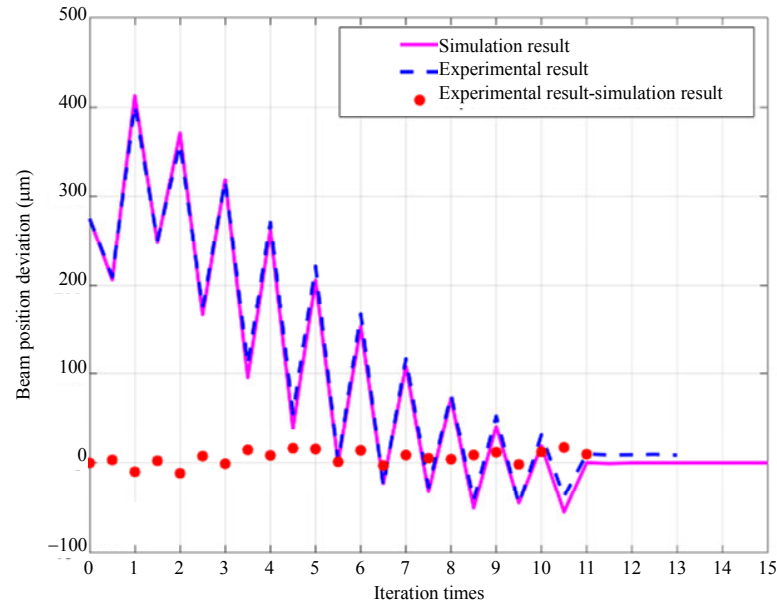

(c)

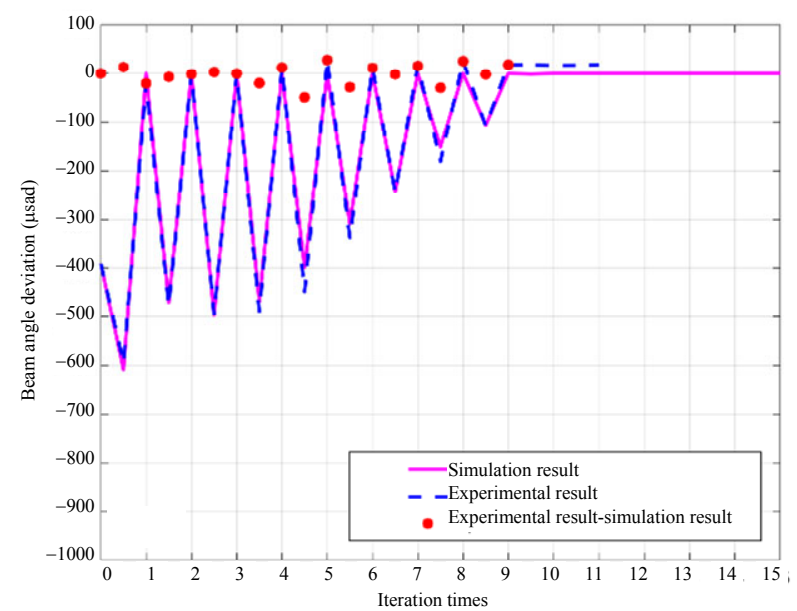

(b)

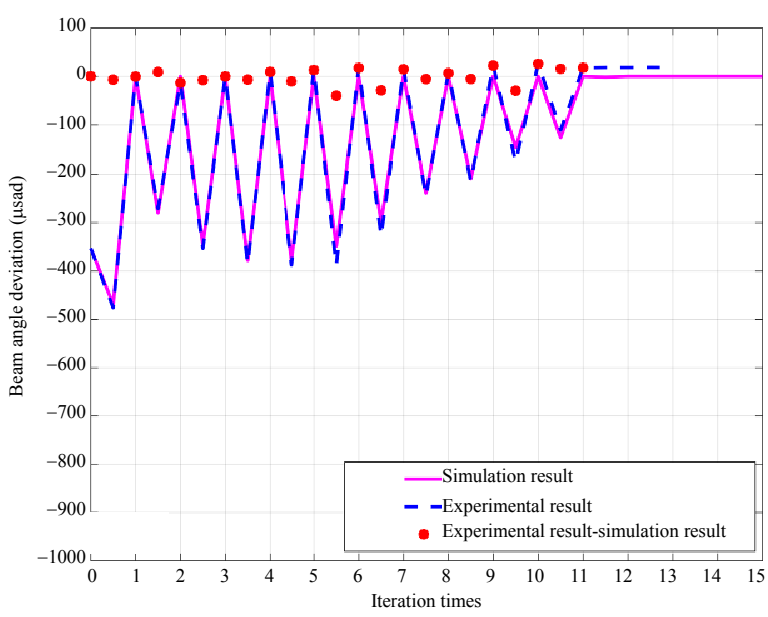

(d)

Fig. 5 Experimental and simulation results for the changes in the beam position and angle deviation with the PLW iterative algorithm: (a) and (b) show the beam position and angle deviations with parameters \#1, respectively, (c) and (d) show the beam position and angle deviations with parameters \#2, respectively. 
We choose another group of parameters for the PLW iterative algorithm. The parameters are $m=2$, $\xi_{a, 1}=250 \mu \mathrm{rad}, \xi_{a, 2}=150 \mu \mathrm{rad}, k_{p, \min }=0.25, k_{p, \max }=$ $D_{1} /\left(D_{1}-D_{2}\right)-0.05=3.88, \Delta_{1}=0.15$, and $\Delta_{2}=0.4$ (parameters \#2). The changes in the position and angle deviations are shown in Figs. 5(c) and 5(d). Compared with Figs. 5(a) and 5(b), taking a smaller value of $k_{p}$, min leads to a lower angle overshoot. Nevertheless, the convergence becomes slower as the iteration times are increased to 11 in Figs. 5(c) and 5(d). As shown in Figs. 4 and 5, changes in $k_{p}$ have little influence on the position overshoot $p_{(1,2)}=$ $m_{p} p_{0}-D_{2} a_{0}$. This is because $m_{p}=1-\left(D_{1}-D_{2}\right) k_{p} / D_{1}$ is close to 1 for $k_{p \text {, min }}$ is small in the first iteration.

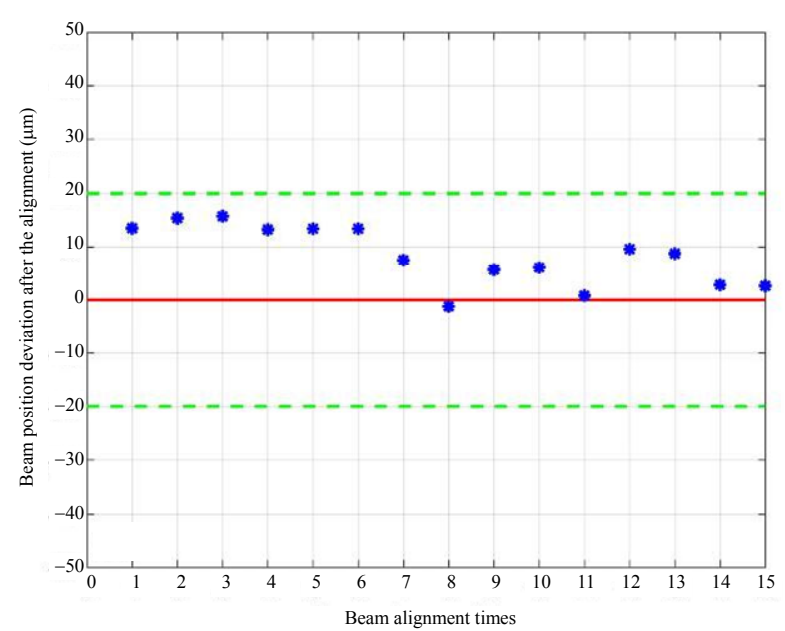

(a)

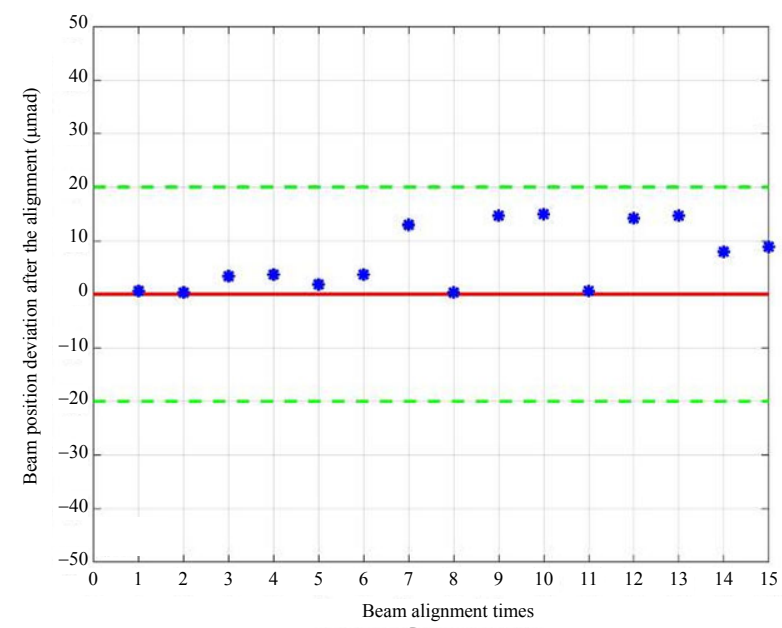

(b)

Fig. 6 Beam position and angle deviations after the alignment with the PLW iterative algorithm in 15 repeated experiment: (a) and (b) show the beam position and angle deviations, respectively.
The beam alignment repeats 15 times with parameter \#1 to verify the effectiveness of the PLW iterative algorithm. The beam position and angle deviations after the alignment are shown in Fig. 6. They all meet the alignment tolerance of $20 \mu \mathrm{rad}$ (angle) and $20 \mu \mathrm{m}$ (position). The repeatability is $5.514 \mu \mathrm{m}(\sigma$, position) and $6.002 \mu \mathrm{rad}(\sigma$, angle $)$, respectively. The nonlinearity of the picomotors, the small differences in the initial deviation and the environmental changes can affect the repeatability.

A large initial deviations $a_{0}=-1000 \mu \mathrm{rad}$ and $p_{0}=$ $1750 \mu \mathrm{m}$ are chosen for simulation with the same optical parameters and tolerances as those in the experiment. Table 1 shows the parameters and simulation results. Compared with the original iterative algorithm, the combined two-stage weighted and PLW iterative algorithm both can reduce the angle overshoot in the same degree with the position overshoot increasing slightly when $k_{p}$ 's in the first iteration are equal. The convergence speed of the PLW iterative algorithm is faster than the combined two-stage weighted iterative algorithm. That means the PLW iterative algorithm gives consideration to both of the overshoot suppression and the convergence speed. The simulation results agree with those in the experiment.

As shown in Fig. 2(b), the position changes of the regulating beam on the PDP can be roughly observed by the spot on the screen with the naked eye during the alignment process, but the spot movement can't be observed clearly due to the limited resolution of the eye and the stray light of the spot, especially in the posterior parts of the alignment. Nevertheless, the coincidence of the spots of the reference light and the regulating light on the screen still can be checked after alignment. Figure 7(a) shows the photo of the spots on the screen before alignment with a large initial deviation in order to distinguish clearly $\left(a_{0}\right.$ and $p_{0}$ in the $X$ direction are $-1019 \mu \mathrm{rad}$ and $1744 \mu \mathrm{m}$, respectively. $a_{0}$ and $p_{0}$ in the $Y$ direction are $-2131 \mu \mathrm{rad}$ and $1662 \mu \mathrm{m}$, respectively). The spot of the reference 
beam is shown in Fig. 7(b). Figure 7(c) shows the spot of the regulating beam after the beam alignment in $X$ and $Y$ directions. The weaker intensity and smaller diameter are caused by the characteristic difference of the mirrors and the beam splitters. As shown in Fig. 7(d), the two spots are superposed when S2 is removed after the alignment, which is an assisted proof for the beam alignment.

Table 1 Parameters for the simulation and simulation results

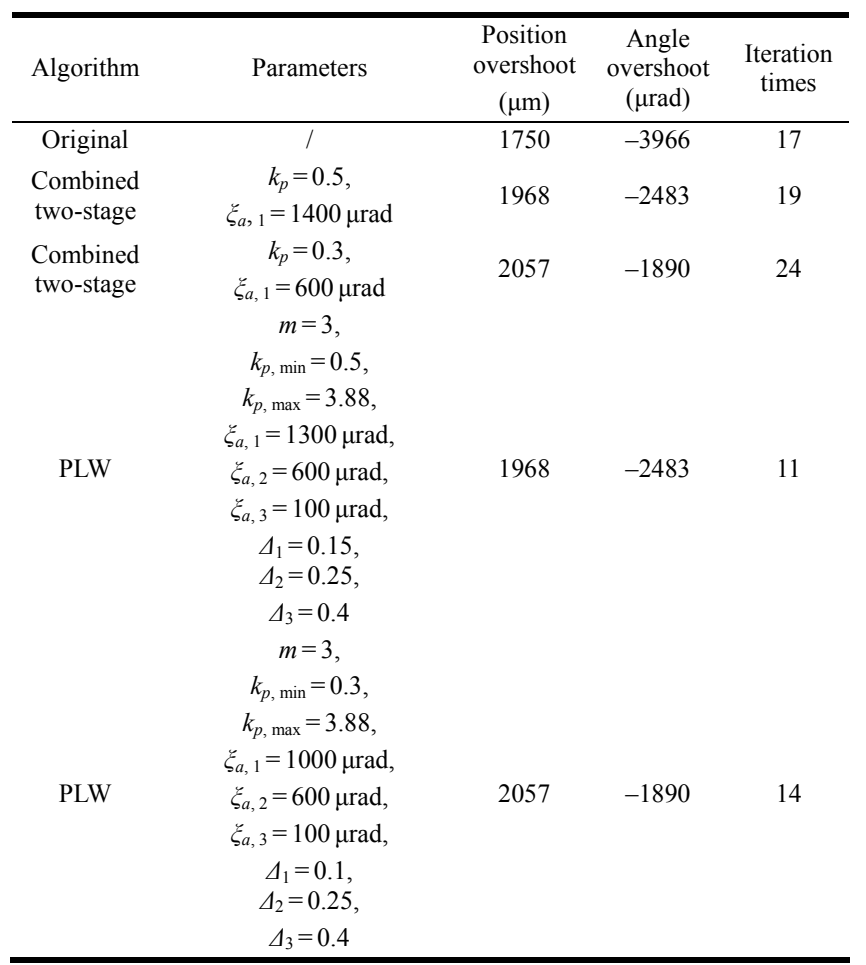
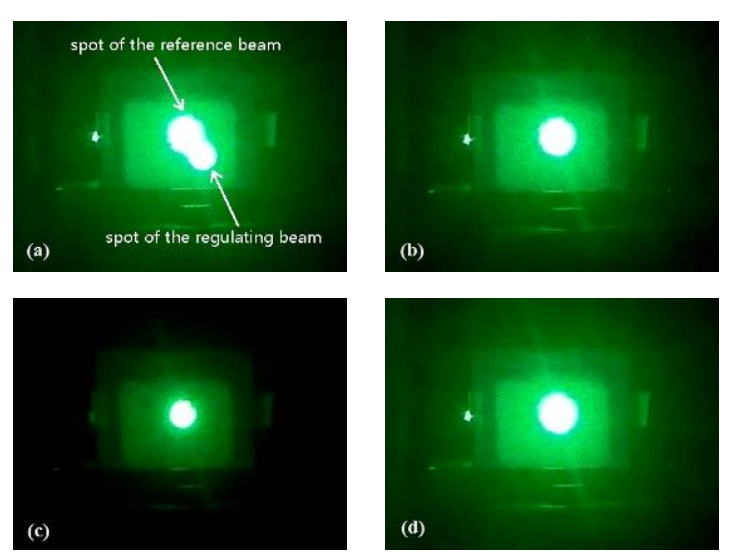

Fig. 7 Spots of the reference beam and regulating beam on the screen: (a) shows the spots before the alignment, (b) shows the spot of the reference beam on the screen in the reference beam path, (c) shows the spot of the regulating beam after the beam alignment in the $X$ and $Y$ directions, and (d) shows the overlapping spots when $\mathrm{S} 2$ are removed after the alignment.

\section{Discussion}

As shown in (4), $m_{p}{ }^{(n-1)}$ represents the convergence speed of the weighted iterative algorithm when $k_{a}=1$. According to the experimental parameters, the changes in $m_{p}{ }^{(n-1)}$ with different iterative algorithms are shown in Fig. 8. The original iterative algorithm has a consistent convergence speed during the whole alignment process. The combined two-stage weighted algorithm converges slowly at the beginning of the alignment to decrease the angle overshoot. Next, the convergence speed is the same as that with the original algorithm. The whole alignment process displays a lower speed. The convergence speed of the PLW iterative algorithm at the beginning is decided by $k_{p \text {, min }}$, which is usually small to obtain a small overshoot. In the middle part of the alignment, the convergence is gradually accelerated. Once the angle deviation is smaller than a certain threshold, the iteration converges as fast as possible with a large $k_{p, \text { max }}$ value. The PLW iteration algorithm can simultaneously give rise to a low overshoot and high-speed convergence.

As shown in Section 4, choosing different parameters in the PLW iterative algorithm can change $k_{p}$ in different ways, and the angle overshoot and convergence speed will vary correspondingly. We discuss the parameter selection method theoretically and by simulation. In this instance, we take $k_{p}$ regulation in the $X$ direction for illustration too.

Firstly, the maximum value for $k_{p}$, min can be determined by the angle overshoot $a_{(1,1)}$. Assume that the value for the $X$ coordinate of the angle alignment target on the APSD is $x_{a, r}$. The change in the $X$ coordinate on the APSD in the first iteration is $K_{\text {angle }} a_{(1,1)}+x_{a, r}$, which must be within the $X$ coordinate range $\left[x_{a}, \min , x_{a}, \max \right]$ of the APSD. According to the expression of $a_{(1,1)}=-k_{p, \min } p_{0} / D_{1}+$ $a_{0}$, the maximum value for $k_{p \text {, min }}$ can be determined to keep the spot in the range of the APSD at the beginning of the iteration. 


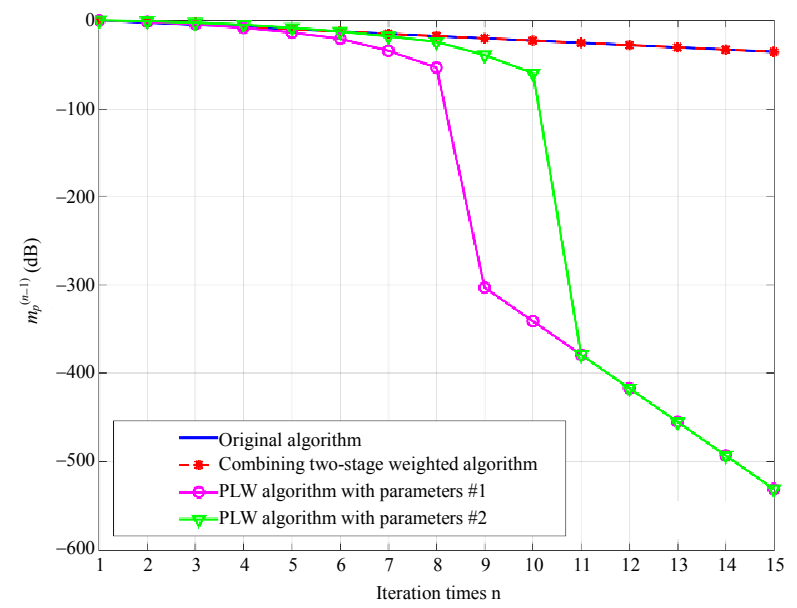

(a)

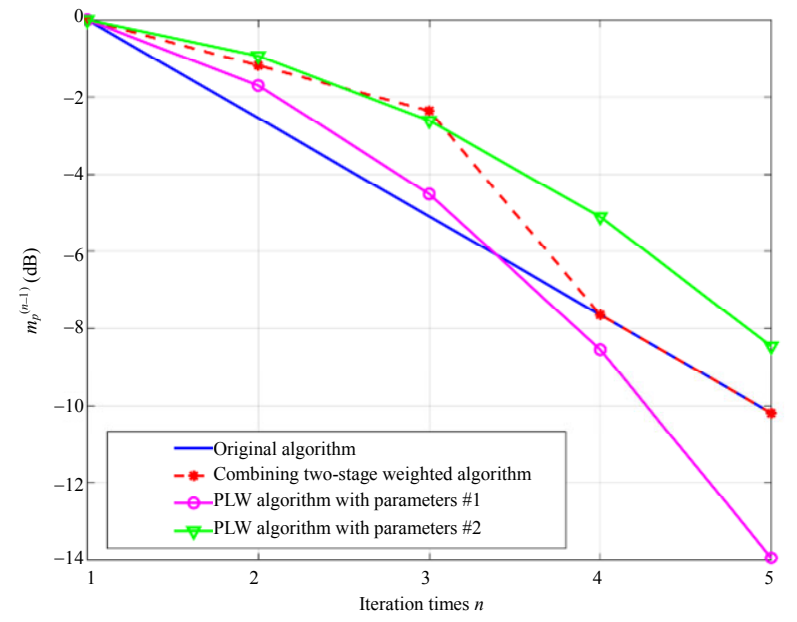

(b)

Fig. 8 Changes in $m_{p}^{(n-1)}$ with different iterative algorithms in the alignment process: (a) shows the changes in $m_{p}^{\left({ }^{(n-1)}\right.}$ in the whole alignment process $(n \leq 15)$ and (b) shows the changes in $m_{p}{ }^{(n-1)}$ at the beginning of the alignment process $(n \leq 5)$.

Secondly, although a small $k_{p \text {, min }}$ can reduce the angle overshoot at the beginning of the alignment, a smaller $k_{p, \min }$ is not better. If $k_{p, \min }$ is smaller, $\Delta_{1}$ should be smaller, and the width of the initial segments should be larger. This means the convergence speed is decreased. Otherwise, a large angle deviation may appear at the middle and posterior parts of the alignment, which may also give rise to alignment failure caused by the measuring spot exceeding the detector range. For example, we choose the same parameters as those in parameters $\# 1$, except for $k_{p \text {, min. }} k_{p \text {, min }}$ is changed from 0.5 to 0.2 . The simulation result indicates that deviations of $-515 \mu \mathrm{rad}$ and $-512 \mu \mathrm{rad}$ appear in the third and fourth iterations, respectively, which are larger than the angle overshoot $-488 \mu \mathrm{rad}$ in the first iteration. Therefore, choosing the parameters of the PLW iterative algorithm is a compromise. To avoid the probable risk of alignment failure in the whole alignment process, a smaller overshoot leads to a slower iteration. Besides, $m, \Delta_{i}$, and $\xi_{a, i}$ are important parameters in the PLW iterative algorithm. The larger of the angle or position overshoot is, the larger $m$ can be chosen to smooth the alignment. Nevertheless, the algorithm becomes more complicated due to an increase in the number of parameters with a larger $m$. As shown in (10), $\Delta_{i}<$ $\Delta_{i+1}$, it means the first order difference of $\Delta_{i}$ is positive. When choosing $\Delta_{i}$ to make the second order difference of $\Delta_{i}$ positive, the convergence speed can increase gradually with a positive acceleration. $\xi_{a, i}$ can be chosen a certain percentage of the angle or position overshoot as needed. Generally, $\xi_{a, m}$ is in the order of hundred $\mu \mathrm{rad}(\mu \mathrm{m})$ to keep the stability at the posterior parts of the alignment.

Thirdly, the small APSD and PPSD placement errors exist in reality. The APSD placement error impacts the accuracy of the decoupled angle readout. Reference [21] analyzed the effect and gave the angle readout error caused by the APSD placement error. It can be described as (12)

$$
\delta a_{e}=\delta a\left[1-\left(1-\frac{L_{\mathrm{pa}}}{f_{2}}\right) \frac{\Delta L_{2}}{f_{2}}+O\left[\frac{\Delta L_{2}}{f_{2}}\right]^{2}\right]
$$

where $\delta a_{e}$ is the angle readout with the APSD placement error $\Delta L_{2}, \delta a$ is the exact angle readout if there is no placement error, and $L_{\mathrm{pa}}$ is the distance from the PDP to the ADP. In our system, $L_{\mathrm{pa}}$ is about $700 \mathrm{~mm}$, and $\Delta L_{2}$ is estimated $2 \mathrm{~mm}$ with careful placement. According to (12), the relative angle readout error is $3.75 \%$, and the maximum readout error is at the edge of the APSD. The PLW iterative algorithm can make the measuring spot away from the edge of PSD. Hence, the angle readout error due to the APSD misplacement is quite small. The PPSD placement error only changes the location of the PDP, which has no influence on the beam alignment. 
Fourthly, as the simulation results have a high coherence with the experimental results, the simulation program can be embedded in an automatic alignment program. Before the alignment, the measured $a_{0}$ and $p_{0}$ are input to the simulation program, and the parameters of the PLW iterative algorithm are set. Then, the alignment processes are simulated to check the performance of the parameters in the program online. The online simulation technique can optimize parameters for practical applications. It is cost effective and time saving.

The position overshoot can be reduced by regulating $k_{a}$. The situations in the $Y$ direction are similar. Thus, we do not discuss them.

\section{Conclusions}

We have demonstrated a new PLW iterative algorithm for beam alignment in SBIL. The convergence property of the general weighted iterative algorithm model is analyzed mathematically, with the ranges of the weight factors determined. In the PLW iterative algorithm, the key weight factor impacting the large overshoot the most is changed linearly piecewise within the value range, with the other weight parameter being kept at a constant value of 1 . The changes in angle and position deviations during alignment with different iterative algorithms are experimented and simulated for comparison. The experimental and simulation results show good consistency, which indicates that the PLW iterative algorithm can reduce the angle or position overshoot at the beginning of the alignment. The probable alignment failure caused by exceeding the detector range can be avoided. Beyond that, the convergence speed can be effectively raised compared with the previous algorithm. The algorithm proposed can be applied to accomplish beam alignment in SBIL both reliably and quickly.

\section{Acknowledgment}

The authors thank Dr. Wenhao LI, Zhaowu LIU, and Zhihong BAI for their useful suggestion and assistance.

The research was supported by the National Natural Science Foundation of China (NSFC) (Grant No. 61227901) and Jilin Province Science \& Technology Development Program Project in China (Grant No. 20190103157JH).

Open Access This article is distributed under the terms of the Creative Commons Attribution 4.0 International License (http://creativecommons.org/licenses/by/4.0/), which permits unrestricted use, distribution, and reproduction in any medium, provided you give appropriate credit to the original author(s) and the source, provide a link to the Creative Commons license, and indicate if changes were made.

\section{References}

[1] N. A. Elmahdy, M. S. Esmail, and M. M. El-Okr, "Characterization of a thermal sensor based on one-dimensional photonic crystal with central liquid crystal defect," Optik, 2018, 170: 444-451.

[2] K. C. Tseng, S. T. Hong, T. H. Lin, T. H. Chuang, and C. C. Fu, "WGP structures patterned by Lloyd's mirror laser interference lithography system integrate into MEMS physical sensor device," SPIE, 2016, 9759: 97591E-1-97591E-6.

[3] M. C. Gupta, C. Ungaro, J. J. Foler IV, and S. K. Gray, "Optical nanostructures design, fabrication, and application for solar/thermal energy conversion," Solar Energy, 2018, 165: 100-114.

[4] K. Lee, J. Lee, B. A. Mazor, and S. R. Forrest, "Transforming the cost of solar-to-electrical energy conversion: integrating thin-film GaAs solar cells with non-tracking mini-concentrators," Light: Science \& Applications, 2015, 4: e288-1-e288-7.

[5] A. M. Shuty, S. V. Eliseeva, and D. I. Sementsov, "Dynamics of the magnetic nanoparticles lattice in an external magnetic field," Journal of Magnetism and Magnetic Materials, 2018, 464: 76-90.

[6] M. Gu, X. P. Li, and Y. Y. Gao, "Optical storage arrays: a perspective for future big data storage," Light: Science \& Applications, 2014, 3: e177-1-e177-11.

[7] J. Yuan, Y. Y. Xie, Z. X. Geng, C. X. Wang, H. M. Chen, Q. Kan, et al., "Enhanced sensitivity of gold elliptic nanohole array biosensor with the surface plasmon polaritons coupling," Optical Materials Express, 2015, 5(4): 818-826.

[8] Q. S. Li, L. Cai, Y. H. Ma, J. H. Yang, Y. Yang, Q. J. Meng, et al., "Research progress of biosensors based on long period fiber grating," Chinese Optics, 2018, 
11(3): 476-502.

[9] C. H. Liu, M. H. Hong, M. C. Lum, H. Flotow, F. Ghadessy, and J. B. Zhang, "Large-area micro/nanostructures fabrication in quartz by laser interference lithography and dry etching," Applied Physics A, 2010, 101(2): 237-241.

[10] A. Retolaza, A. Juarros, J. Ramiro, and S. Merino, "Thermal roll to roll nanoimprint lithography for micro pillars fabrication on thermoplastics," Microelectronic Engineering, 2018, 193: 54-61.

[11] Y. Kanamori, M. Okochi, and K. Hane, "Fabrication of antireflection subwavelength grating at tips of optical fibers using UV nanoimprint lithography," Optics Express, 2013, 21(1): 322-328.

[12] Y. F. Chen, "Nanofabrication by electron beam lithography and its application: a review," Microeletronic Engineering, 2015, 135: 57-72.

[13] N. L. Chiromawa and K. Lbrahim, "Fabrication of micro-array of Fresnel rings on Si by electron beam lithography and reactive ion etching," Applied Physics A, 2016, 122(2): 122-129.

[14] K. L. Jim, C. W. Leung, and H. L. W. Chan, "Photonic crystal cavity embedded barium strontium titanate thin-film rib waveguide prepared by focused ion beam etching," Thin Solid Films, 2010, 518(24): e101-e103.

[15] J. Liu, T. Jia, K. Zhou, D. Feng, S. Zhang, H. Zhang, et al., "Direct writing of $150 \mathrm{~nm}$ gratings and squares on $\mathrm{ZnO}$ crystal in water by using $800 \mathrm{~nm}$ femtosecond laser," Optics Express, 2014, 22(26): 32361-32370.

[16] S. M. Jing, X. Y. Zhang, J. F. Liang, C. Chen, Z. M. Zheng, and Y. S. Yu, "Ultrashort fiber Bragg grating written by femtosecond laser and its sensing characteristics," Chinese Optics, 2017, 10(4): 449-454.

[17] D. X. Liu, H. Xia, Y. L. Sun, Q. D. Chen, and W. F. Dong, "Femtosecond laser direct writing bio-gel template for in situ synthesis of nanoparticles,"
Chinese Optics, 2014, 7(4): 608-615.

[18] J. Montoya, "Toward nano-accuracy in scanning beam interference lithography," Ph.D. dissertation, Massachusetts Institute of Technology, Boston, USA, 2006.

[19] W. Wei, "Study on beam quality control of the scanning beam interference lithography system," $\mathrm{Ph} . \mathrm{D}$. dissertation, University of Chinese Academy of Sciences, Beijing, China, 2017.

[20] P. T. Konkola, C. G. Chen, R. K. Heilmann, and M. L. Schattenburg, "Beam steering system and spatial filtering applied to interference lithography," Journal Vacuum Science \& Technology B, 2000, 18(6): 3282-3286.

[21] C. G. Chen, "Beam alignment and image metrology for scanning beam interference lithography: fabricating gratings with nanometer phase accuracy," Ph.D. dissertation, Massachusetts Institute of Technology, USA, 2003.

[22] W. Wang, Bayanheshig, Y. Song, S. Jiang, and M. Z. Pan, "Beam alignment and convergence analysis of scanning beam interference lithography system," Chinese Journal of Lasers, 2016, 43(12): 176-183.

[23] T. Kanai, A. Suda, S. Bohman, M. Kaku, S. Yamaguchi, and K. Midorikawa, "Pointing stabilization of a high-repetition-rate high power femtosecond laser for intense few-cycle pulse generation," Applied Physics Letters, 2008, 92(6): 061106-1-061106-3.

[24] A. Stalmashonak, N. Zhavoronkov, I. V. Hertel, S. Vetrov, and K. Schmid, "Spatial control of femtosecond laser system output with submicroradian accuracy," Applied Optics, 2006, 45(6): 1271-1274.

[25] Y. Song, W. Wang, S. Jiang, Bayanheshig, and N. Zhang. "Weighted iterative algorithm for beam alignment in scanning beam interference lithography," Applied Optics, 2017, 56(31): 8669-8675. 